\title{
Sentinel Node Detection in Patients with Thyroid Carcinoma: A Meta-analysis
}

\author{
P. G. H. M. Raijmakers • M. A. Paul • \\ P. Lips
}

Published online: 2 July 2008

(C) The Author(s) 2008

\begin{abstract}
Objective This study was designed to review the diagnostic performance of sentinel node (SN) detection for assessment of the nodal status in thyroid carcinoma patients and to determine the technique (using blue dye or Technetium-99m colloid $\left({ }^{99 m} \mathrm{Tc}\right)$ ) that demonstrated the highest success rate with regard to the detection rate and sensitivity.

Methods A comprehensive computer literature search of studies published in English language through December 2007 and regarding SN procedures in patients with thyroid disorders was performed in MEDLINE. Pooled values regarding the $\mathrm{SN}$ detection rate and the pooled sensitivity values of the SN procedure were presented with a $95 \%$ confidence interval (CI) for the different SN detection techniques.

Results Ultimately, we identified 14 studies comprising a total of 457 patients. Of these, ten studies $(n=329$ patients) used the blue dye technique with a pooled SN detection of $83 \%$ (95\% CI, 79-87\%). The remaining four
\end{abstract}

P. G. H. M. Raijmakers ( $\square)$

Department of Nuclear Medicine \& PET Research,

VU University Medical Center, Amsterdam, The Netherlands

e-mail: p.raijmakers@vumc.nl

P. G. H. M. Raijmakers

Department of Clinical Epidemiology and Biostatistics,

VU University Medical Center, Amsterdam, The Netherlands

M. A. Paul

Department of Surgery, VU University Medical Center,

Amsterdam, The Netherlands

P. Lips

Department of Endocrinology, VU University Medical Center,

Amsterdam, The Netherlands studies $(n=128)$ used ${ }^{99 \mathrm{~m}}$ Tc-colloid with a pooled $\mathrm{SN}$ detection of $96 \%$ (95\% CI, 91-99\%; $p<0.05$ vs. blue dye technique).

Conclusion In patients with suspected thyroid carcinoma, SN biopsy demonstrated a higher SN detection rate when ${ }^{99 \mathrm{~m}} \mathrm{Tc}$ was used $(96 \%)$ instead of the blue dye technique $(83 \%)$.

\section{Introduction}

Lymph node involvement is commonly detected in patients with thyroid cancer, reportedly up to $70 \%$ [1-3]. Furthermore, cervical lymph node metastases are a frequent cause of recurrent thyroid cancer [4]. However, the prognostic importance and role of elective lymphadenectomy in thyroid cancer is controversial. Nodal metastases are a significant risk factor for survival in patients with thyroid cancer [3]. Because this risk varies considerably between different age groups, the prognostic importance and role of elective lymphadenectomy are controversial. Hence, knowledge regarding the presence or absence of lymphatic metastases may be of interest in the staging procedure of patients with thyroid cancer. Systematic lymphadenectomy is a standard technique, which is currently used to detect lymphatic metastatic spread. Alternative techniques, including resection of macroscopic enlarged nodes, also are used. Heterogeneity with regard to removal of the lymph nodes may introduce bias during the staging of thyroid patients, thereby affecting the predictive value of staging classification procedures, including the presence of lymphatic metastatic spread. Furthermore, this heterogeneity in the lymph node removal may explain the controversy regarding the optimal procedure. Certain institutions 
perform routine lymph node dissection, whereas others refrain from routine dissection.

Recent studies suggested the use of sentinel node (SN) detection as a less invasive method for studying the lymphatic metastatic spread in patients with breast cancer, melanoma, cervical cancer, and thyroid carcinoma [5-8]. However, considerable variation has been reported between studies with regard to the success rates of these $\mathrm{SN}$ procedures in patients with thyroid cancer; for example, Dixon et al. reported SN detection in merely $65 \%$ of the patients, whereas others have reported $100 \%$ detection rates $[9,10]$. In a recently concluded meta-analysis regarding SN detection in patients with cervical cancer, we observed that the use of radiotracers vs. the blue dye technique was an important factor that determined the success rate of SN detection [11].

This review and meta-analysis was designed to determine whether sentinel lymph nodes (SLNs) can be identified accurately in patients with thyroid cancer with the injection of a radiotracer or a blue dye. Furthermore, we studied the effect of different detection techniques on the $\mathrm{SN}$ detection rate in these patients.

\section{Methods}

\section{Study selection}

A comprehensive computer literature search of studies published in English language and comprising human subjects was performed to identify articles related to the diagnostic performance of $\mathrm{SN}$ and thyroid carcinoma when compared with histopathological findings used as the reference standard.

MEDLINE database through December 2007 was searched for the following terms: "thyroid neoplasm"; "sentinel" as the medical subject headings (MeSH). The list of articles was supplemented by cross-checking the reference lists containing the identified articles. Review articles, letters, comments, conference proceedings, unpublished data, and case reports were excluded from this review.

We included studies that reported SN detection using the blue dye technique or a radiotracer in patients with suspected thyroid carcinoma. We used the above-mentioned inclusion and exclusion criteria [12, 13]. In short, only those studies that satisfied all of the following criteria were included: 1) minimum sample size of ten included patients; 2) the use of a radioactive tracer and/or blue dye during the SN procedure; 3 ) presence of "gold standard," which was defined by the use of histopathological evaluation of surgically resected specimens, for sensitivity analysis; and 4) sufficient data to reassess sensitivity (number of true positives and false negatives) or the $\mathrm{SN}$ detection rate. The exclusion criterion was an overlap in the patient data (duplicate publication). In such cases, the more recent article with most patients was included with an adequate description of the study population.

Data analyses

The sensitivity of the SN procedure was determined from the number of true-positive (TP) and false-negative (FN) results obtained from individual studies. Studies that did not present patients with tumor-positive SNs were excluded from statistical pooling for the sensitivity analyses. However, these studies were included for pooling of the SN detection rate. Heterogeneities in the sensitivity and SN detection rate were tested by using the $\chi^{2}$ test. Additionally, we performed subgroup analysis for the different SN detection techniques: one group comprised studies that used ${ }^{99 \mathrm{~m}} \mathrm{Tc}$-colloid, and another group comprised studies that used the blue dye technique for SN detection. In the event of persisting heterogeneity between studies despite the subgroup analysis, we used the random effect model for statistical pooling of the data; on the other hand, a fixed effect model was implemented in the event of homogeneity. Pooled data are presented with $95 \%$ confidence intervals (CI). Finally, a Z-test was used to compare the pooled estimates of the values of sensitivity and $\mathrm{SN}$ detection rate obtained from the different $\mathrm{SN}$ detection techniques (blue dye or ${ }^{99 \mathrm{~m}}$ Tc-colloid). $P<0.05$ was considered significant.

\section{Results}

Fourteen studies regarding SN were included to assess the SN detection rate in patients with a suspected thyroid carcinoma [9, 10, 14-25]. The present review comprised a total of 457 patients (Table 1). An intratumoral injection technique was used in the majority of the studies (10 of 14 studies), whereas a peritumoral injection technique was used in only four studies (Table 2).

There was considerable variance in the histopathological procedure among the studies included for the analyses. For example, eight studies mentioned the use of an immunohistochemistry (IHC) technique for lymph node evaluation. Of these, two studies implemented the IHC technique in the SN only, whereas the remaining studies implemented IHC in all the lymph nodes. Furthermore, only two studies described the number of sections or slice thickness prepared during the histopathological analysis.

Based on the technique used for SN detection, the studies were divided into the following two groups: four studies ( $n=128$ patients) used the ${ }^{99 \mathrm{~m}} \mathrm{Tc}$-colloid technique; and ten studies ( $n=329$ patients) used the blue dye technique for the purpose of SN detection. The pooled SN detection 
Table 1 Patient characteristics of the studies included for the present meta-analysis

\begin{tabular}{|c|c|c|c|c|c|c|}
\hline Study & Year & $n$ & $\begin{array}{l}\text { Male/female } \\
\text { ratio }\end{array}$ & $\begin{array}{l}\text { Mean age } \\
\text { (yr) [range] }\end{array}$ & $\begin{array}{l}\text { Thyroid nodule } \\
\text { (\% malignant) }\end{array}$ & $\begin{array}{l}\text { Mean tumor } \\
\text { size }(\mathrm{cm}) \text { [range] }\end{array}$ \\
\hline Dixon et al. [9] & 2000 & 40 & $5 / 35$ & $42[25-75]$ & 33 & {$[1-3.5]$} \\
\hline Kelemen et al. [18] & 1998 & 17 & $3 / 14$ & $48[22-68]$ & 71 & $2[0.8-4]$ \\
\hline Haigh and Giuliano [19] & 2000 & 38 & - & - & 45 & - \\
\hline Arch-Ferrer et al. [20] & 2001 & 22 & $2 / 20$ & 37 & 100 & 2.5 \\
\hline Takami et al. [21] & 2003 & 68 & $8 / 60$ & 42 [22-69] & 100 & $1.9[0.6-3.9]$ \\
\hline Tsugawa et al. [22] & 2001 & 38 & $8 / 30$ & $48[24-72]$ & 100 & $1.3[0.5-2.8]$ \\
\hline Pelizzo et al. [16] & 2001 & 29 & $8 / 21$ & $44[21-86]$ & 100 & $1.8[0.5-4]$ \\
\hline Chow et al. [23] & 2004 & 15 & $3 / 12$ & 54 median [26-71] & 100 & - \\
\hline Fukui et al. [24] & 2001 & 22 & $3 / 19$ & 53 & 100 & 1.3 \\
\hline Dzodic et al. [15] & 2006 & 40 & $10 / 30$ & $42[23-57]$ & 100 & 1.5 [median] \\
\hline Sahin et al. [25] & 2001 & 13 & - & {$[30-49]$} & 39 & - \\
\hline Stoeckli et al. [10] & 2003 & 10 & $2 / 8$ & $50[20-74]$ & 67 & - \\
\hline Pelizzo et al. [17] & 2006 & 41 & $12 / 29$ & $40[21-68]$ & 98 & $1.24[0.5-3]$ \\
\hline Carcoforo et al. [14] & 2007 & 64 & $7 / 57$ & 50 & 92 & 0.87 \\
\hline
\end{tabular}

rate for studies using blue dye was 0.83 (95\% CI, 0.79-0.87) and it was $0.96(95 \% \mathrm{CI}, 0.91-0.99 ; p<0.05)$ for studies using the ${ }^{99 \mathrm{~m}}$ Tc-colloid (Fig. 1). Hence, the difference of detection rates was reaching a significant level and there was no overlap of the confidence intervals. Pooling of the sensitivity was performed for a total of six studies, which used the blue dye technique for SN detection; the pooled sensitivity was 0.873 (95\% CI, 0.79-0.93). Accordingly, the false-negative rate was $12.7 \%$. One of the four studies using ${ }^{99 \mathrm{~m}} \mathrm{Tc}$-colloid reported a false-negative rate of $11.3 \%$.

\section{Discussion}

The results of this meta-analysis indicate that studies using the technetium-labeled colloid ( ${ }^{99 \mathrm{~m}} \mathrm{Tc}$-colloid) technique yielded an approximately $13 \%$ higher SN detection rate compared with those using the blue dye technique for $\mathrm{SN}$ detection. Hence, detection of $\mathrm{SNs}$ in patients with suspected thyroid carcinoma using a technetium-labeled colloid may be more effective compared with that obtained with the use of the blue dye technique. There are little data regarding the false-negative rate (FNR) for SN detection with the use of ${ }^{99 m}$ Tc-colloid, only one study reported a FNR of $11.3 \%$ [14]. In contrast, the FNR for SN detection with the use of the blue dye technique was $12.7 \%$. There was no meta-analysis possible of studies that used a combined technique of both blue dye and ${ }^{99 \mathrm{~m}}$ Tc-colloid.

Presently, the surgical options for patients with thyroid carcinoma include total thyroidectomy or unilateral lobectomy. In addition, lymph node sampling may include no sampling, blind sampling, node picking of enlarged lymph nodes, and lymph node dissection. Obviously, differences in the surgical approaches may result in heterogeneity in the tumor classification. SN biopsy is a minimally invasive approach, and it offers a more standardized technique of lymph node assessment. Greater standardization in the evaluation technique renders more robustness to the prognostic value of lymph node metastasis in thyroid cancer patients. Furthermore, it may prompt a change in therapy in certain cases. For example, in institutions using unilateral lobectomy for the surgical treatment of small papillary thyroid carcinomas, the SN technique may offer additional prognostic value. Using the $\mathrm{SN}$ technique, it is possible to identify patients with lymph node metastasis. These patients with small carcinomas and SN metastasis may require total thyroidectomy followed by postoperative 131-iodide therapy (I-131 therapy) instead of unilateral lobectomy without any further treatment.

$\mathrm{SN}$ evaluation also might result in an alteration in the tumor staging of certain patients. Especially, introduction of the IHC technique for detection of tumor cells in the SNs might increase the number of patients with lymph node metastases. With the use of IHC, it is now possible to detect micrometastases, which would have remained undetected earlier [26]; thus, a more accurate evaluation regarding the nodal status can be performed. The detection of micrometastases using SNs may enable selection of patients for further surgery or I-131 therapy; however, it remains to be seen whether additional treatment, such as I-131, would improve survival in patients with single SN micrometastasis. More importantly, lymph node metastasis is not uncommon in patients with small thyroid carcinomas. Noguchi et al. [3] observed that $69 \%$ of the patients with thyroid lesions measuring $<1 \mathrm{~cm}$ demonstrated nodal metastasis. Prospective studies are required to resolve the 


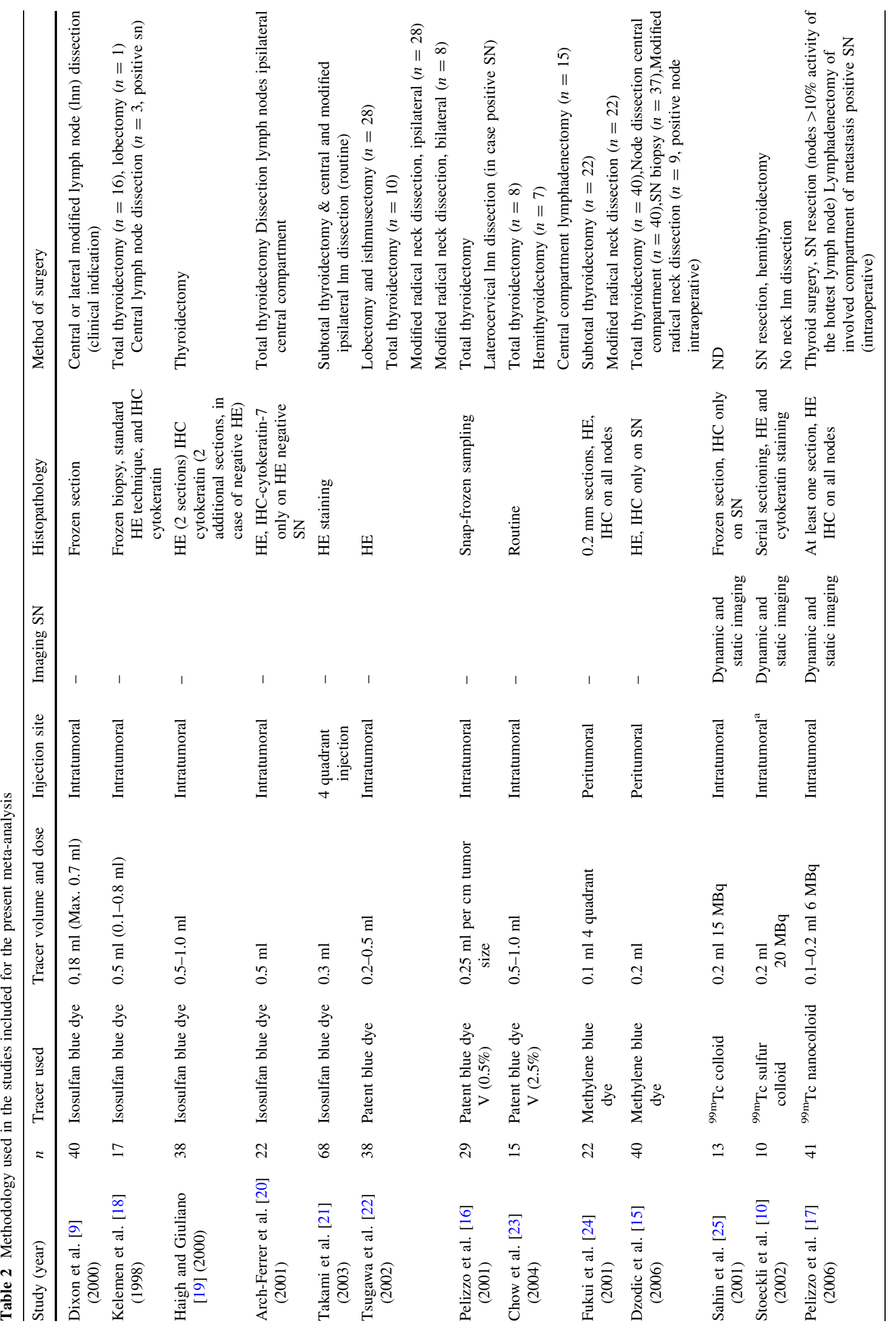


issue regarding the optimal treatment for patients with small papillary thyroid carcinoma and lymph node metastasis.

To facilitate a better prediction regarding the outcome for patients with thyroid cancer, several tumor staging classifications have been proposed. Furthermore, an accurate staging permits optimal stratification for new clinical studies. The National Thyroid Cancer Treatment Cooperative Study (NTCTCS) staging classification registration of 1,607 patients was prospectively validated and showed relatively high accuracy in predicting the outcome of patients with thyroid cancer [27]. According to this staging classification, cervical lymph node metastases are of importance in classifying patients. Patients older than aged 45 years with cervical lymph node metastases were classified as stage III (high risk), whereas those younger than aged 45 years and demonstrating cervical lymph node metastases were considered as stage I (low risk) [27].

There are potential advantages of the SN technique, for example, reduced morbidity compared with elective lymph node dissection. Furthermore, it offers a standardized technique of lymph node evaluation. Although we reviewed a limited number of studies with a total of 457 patients, it seems that the use of ${ }^{99 \mathrm{~m}} \mathrm{Tc}$-colloid enabled identification of SN in an additional 13\% of the patients compared with those identified with the blue dye technique. Hence, for institutions considering the application of SN detection in patients with thyroid carcinoma, the use of ${ }^{99 \mathrm{~m}} \mathrm{Tc}$-colloid for $\mathrm{SN}$ detection offers a potentially higher success rate in identifying SN. The use of blue dye technique for SN detection may be less successful due to transport of the blue dye to the parathyroid glands.

Considering the results of earlier techniques of SN detection in patients with melanoma and breast cancer, the combination of blue dye and ${ }^{99 \mathrm{~m}} \mathrm{Tc}$-colloid might be a superior alternative. Indeed, Catarci et al. [28] successfully performed SN detection in all six patients using a combination of ${ }^{99 \mathrm{~m}} \mathrm{Tc}$-colloid and blue dye. However, more and larger studies are necessary to assess the potential of the dual tracer approach in increased SN detection. Because we did not find any additional evidence regarding this method in the present literature, we could not perform a meta-analysis of this method.

Meta-analysis has certain limitations, and publication bias is a potential confounder. SN studies with negative results (low detection rates or high FNRs may have a lower publication rate compared with those with clearly positive results). Furthermore, the methodological quality varies amongst studies. For example, only 7 of the 14 studies that were included in this meta-analysis have reported FNR data. Future studies reporting SN detection in patients with thyroid cancer should include FNR data in their results. The FNR is the single most important quality item for the SN technique. High numbers of false-negative SN renders 
Fig. 1 Plot of individual study values and pooled estimates of SN detection, including $95 \%$ confidence intervals

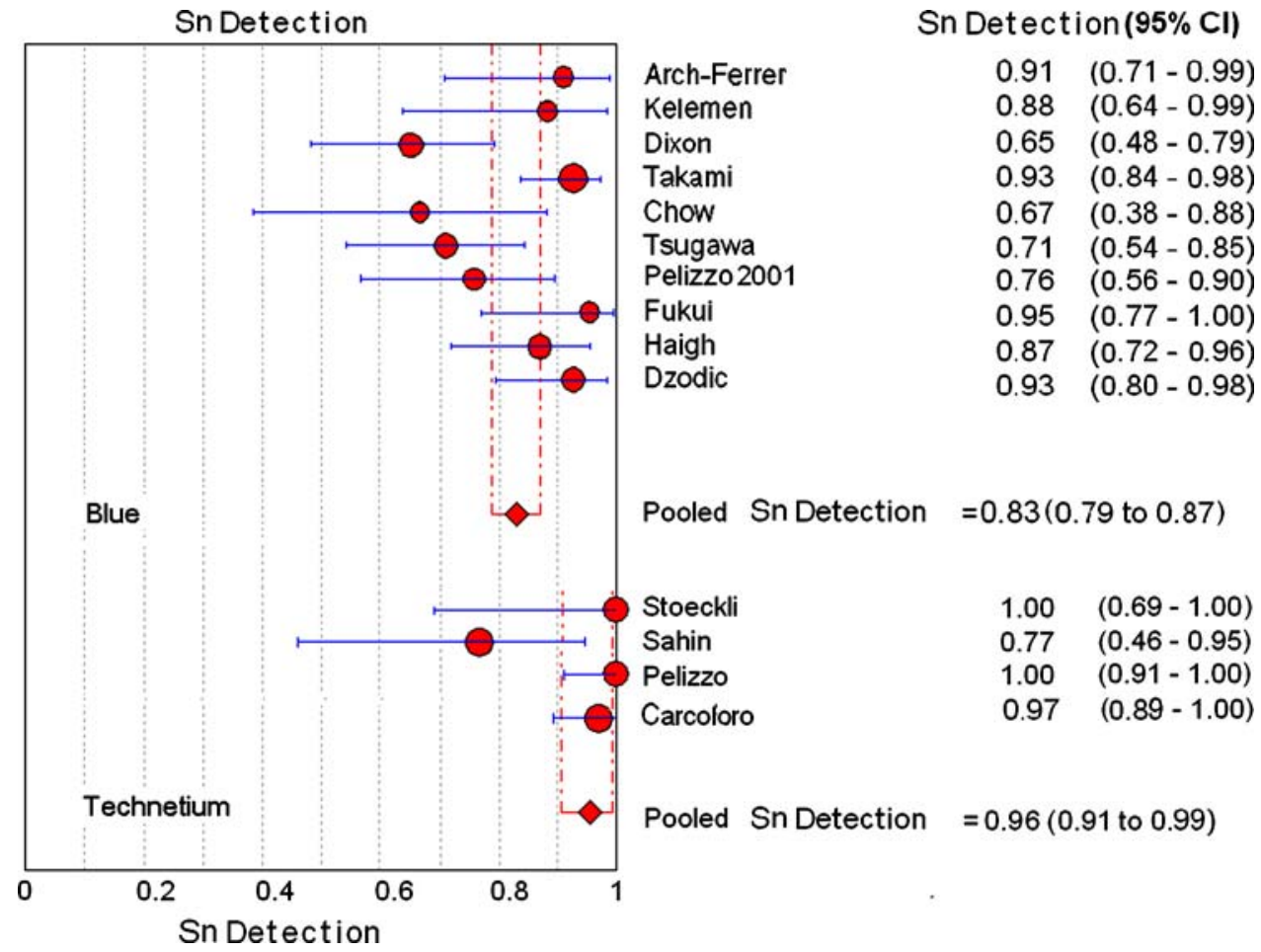

the specific technique unsuitable for routine practice. Indeed, the pooled value of the FNR of six studies that used blue dye is higher compared with those that included patients with breast cancer or melanoma. Hence, further improvements in the SN technique for patients with thyroid carcinoma should be considered before a routine application is established. Future studies should investigate demographic data and data regarding the tumor size should be included to study any potential population differences. Finally, future studies should carefully report the histopathological methods used. It is relatively simple to obtain these data, such as the number of sections through the $\mathrm{SN}$; however, they are lacking in the majority of the studies.

\section{Conclusions}

Our findings indicate that $\mathrm{SN}$ detection is possible in patients with thyroid cancer. The use of ${ }^{99 \mathrm{~m}} \mathrm{Tc}$-colloid enables SN identification in $96 \%$ of the patients. However, the use of blue dye technique is probably associated with a lower SN detection rate compared with that obtained by the ${ }^{99 \mathrm{~m}}$ Tc-colloid technique.

The theoretical advantages of the $\mathrm{SN}$ technique require further probing through clinical studies. Furthermore, more studies are required to identify the optimal SN detection technique, especially with regard to the FNR. Additionally, the clinical impact of SN detection with regard to the prognosis and treatment requires further attention in new clinical studies.
Open Access This article is distributed under the terms of the Creative Commons Attribution Noncommercial License which permits any noncommercial use, distribution, and reproduction in any medium, provided the original author(s) and source are credited.

\section{References}

1. Shaha AR, Shah JP, Loree TR (1996) Patterns of nodal and distant metastasis based on histologic varieties in differentiated carcinoma of the thyroid. Am J Surg 172:692-694

2. Scheumann GF, Gimm O, Wegener G et al (1994) Prognostic significance and surgical management of locoregional lymph node metastases in papillary thyroid cancer. World J Surg 18:559-567

3. Noguchi S, Murakami N, Yamashita H et al (1998) Papillary thyroid carcinoma: modified radical neck dissection improves prognosis. Arch Surg 133:276-280

4. Wanebo HJ, Andrews W, Kaiser DL (1981) Thyroid cancer: some basic considerations. Am J Surg 142:474-479

5. Pijpers R, Collet GJ, Meijer S, Hoekstra OS (1995) The impact of dynamic lymphoscintigraphy and gamma probe guidance on sentinel node biopsy in melanoma. Eur J Nucl Med 22:1238-1241

6. Borgstein PJ, Pijpers R, Comans EF et al (1998) Sentinel lymph node biopsy in breast cancer: guidelines and pitfalls of lymphoscintigraphy and gamma probe detection. J Am Coll Surg 186:275-283

7. Pijpers R, Meijer S, Hoekstra OS et al (1997) Impact of lymphoscintigraphy on sentinel node identification with technetium99m-colloidal albumin in breast cancer. J Nucl Med 38:366-368

8. Pijpers R, Buist MR, van Lingen A et al (2004) The sentinel node in cervical cancer: scintigraphy and laparoscopic gamma probeguided biopsy. Eur J Nucl Med Mol Imaging 31:1479-1486

9. Dixon E, McKinnon JG, Pasieka JL (2000) Feasibility of sentinel lymph node biopsy and lymphatic mapping in nodular thyroid neoplasms. World J Surg 24:1396-1401 
10. Stoeckli SJ, Pfaltz M, Steinert H, Schmid S (2003) Sentinel lymph node biopsy in thyroid tumors: a pilot study. Eur Arch Otorhinolaryngol 260:364-368

11. van de Lande J, Torrenga B, Raijmakers PG et al (2007) Sentinel lymph node detection in early stage uterine cervix carcinoma: a systematic review. Gynecol Oncol 106:604-613

12. Temmerman OP, Raijmakers PG, Berkhof $\mathbf{J}$ et al (2005) Accuracy of diagnostic imaging techniques in the diagnosis of aseptic loosening of the femoral component of a hip prosthesis: a metaanalysis. J Bone Joint Surg Br 87:781-785

13. Temmerman OP, Heyligers IC, Teule GJ et al (2005) The value of contrast and subtraction arthrography in the assessment of aseptic loosening of total hip prostheses: a meta-analysis. Eur $\mathbf{J}$ Radiol 56:113-119

14. Carcoforo P, Feggi L, Trasforini G et al (2007) Use of preoperative lymphoscintigraphy and intraoperative gamma-probe detection for identification of the sentinel lymph node in patients with papillary thyroid carcinoma. Eur J Surg Oncol 33:1075-1080

15. Dzodic R, Markovic I, Inic M et al (2006) Sentinel lymph node biopsy may be used to support the decision to perform modified radical neck dissection in differentiated thyroid carcinoma. World J Surg 30:841-846

16. Pelizzo MR, Boschin IM, Toniato A et al (2001) The sentinel node procedure with Patent Blue V dye in the surgical treatment of papillary thyroid carcinoma. Acta Otolaryngol 121:421-424

17. Pelizzo MR, Merante B I, Toniato A et al (2006) Sentinel node mapping and biopsy in thyroid cancer: a surgical perspective. Biomed Pharmacother 60:405-408

18. Kelemen PR, Van Herle AJ, Giuliano AE (1998) Sentinel lymphadenectomy in thyroid malignant neoplasms. Arch Surg 133: 288-292
19. Haigh PI, Giuliano AE (2000) Sentinel lymph node dissection for thyroid malignancy. Recent Results Cancer Res 157:201-205

20. Arch-Ferrer J, Velazquez D, Fajardo R et al (2001) Accuracy of sentinel lymph node in papillary thyroid carcinoma. Surgery 130: 907-913

21. Takami H, Sasaki K, Ikeda Y et al (2003) Detection of sentinel lymph nodes in patients with papillary thyroid cancer. Asian $\mathrm{J}$ Surg 26:145-148

22. Tsugawa K, Ohnishi I, Nakamura M et al (2002) Intraoperative lymphatic mapping and sentinel lymph node biopsy in patients with papillary carcinoma of the thyroid gland. Biomed Pharmacother 56(Suppl 1):100s-103s

23. Chow TL, Lim BH, Kwok SP (2004) Sentinel lymph node dissection in papillary thyroid carcinoma. ANZ J Surg 74:10-12

24. Fukui Y, Yamakawa T, Taniki $T$ et al (2001) Sentinel lymph node biopsy in patients with papillary thyroid carcinoma. Cancer 92:2868-2874

25. Sahin M, Yapici O, Dervisoglu A et al (2001) Evaluation of lymphatic drainage of cold thyroid nodules with intratumoral injection of Tc-99m nanocolloid. Clin Nucl Med 26:602-605

26. Van Trappen PO, Gyselman VG, Lowe DG et al (2001) Molecular quantification and mapping of lymph-node micrometastases in cervical cancer. Lancet 357:15-20

27. Sherman SI, Brierley JD, Sperling M et al (1998) Prospective multicenter study of thyroid carcinoma treatment: initial analysis of staging and outcome. National Thyroid Cancer Treatment Cooperative Study Registry Group. Cancer 83:1012-1021

28. Catarci M, Zaraca F, Angeloni R et al (2001) Preoperative lymphoscintigraphy and sentinel lymph node biopsy in papillary thyroid cancer. A pilot study. J Surg Oncol 77:21-24 